\title{
A Collocation-Galerkin Method for a First Order Hyperbolic Equation With Space and Time-Dependent Coefficient
}

\author{
By David Archer and Julio César Díaz*
}

\begin{abstract}
A collocation-Galerkin scheme is proposed for an initial-boundary value problem for a first order hyperbolic equation in one space dimension. The Galerkin equations satisfied by the approximating solution are obtained from a weak-weak formulation of the initial-boundary value problem. The collocation points are taken to be affine images of the roots of the Jacobian polynomials of degree $r-1$ on $[0,1]$ with respect to the weight function $x(1-x)$. Optimal $L^{\infty}\left(L^{2}\right)$-norm estimates of the error are derived.
\end{abstract}

1. Introduction. In this paper we propose a collocation-Galerkin scheme for an initial-boundary value problem for a first order hyperbolic equation in one space dimension with a time- and space-dependent coefficient.

Collocation-Galerkin methods were originally introduced by Díaz [3], [4] for the two-point boundary value problem. These methods were introduced in order to define collocation-like schemes for problems in which the local behavior of the solutions was best represented by spaces of lower continuity than required by the collocation scheme. The continuity constraints removed were replaced by conditions on the nodal jumps. It was shown in [3], [4] that certain Galerkin equations impose conditions on certain jumps of the approximating function across the nodes. For a second order problem, the $L^{2}$-Galerkin formulation imposes jump conditions on the first derivative, while the $H^{-1}$-Galerkin formulation imposes conditions on the jumps of both the function and its derivative. For elliptic partial differential equations, Wheeler in [7] has described and analyzed a procedure that uses an interior penalty $L^{2}$-Galerkin formulation to control the jumps across the elements.

In this paper we extend this class of methods to initial-boundary value problems for a first order hyperbolic partial differential equation. The collocation method for this class of problems was described and analyzed by Archer in [1] using continuous piecewise polynomial spaces. The scheme presented here uses collocation on discontinuous approximating spaces. The continuity conditions of the approximating space at the nodes are replaced by Galerkin equations similar to those described by Baker in [2] using a weak-weak formulation of the initial-boundary

Received September 28, 1977; revised March 11, 1980 and April 8, 1981.

1980 Mathematics Subject Classification. Primary 65N30, 65 N35.

* Portions of this work were performed while this author was a visiting assistant professor at the Computer Sciences Department, University of Toronto, Toronto, Canada.

Current address: Mobil Research and Development Corporation, Field Research Laboratory, P.O. Box 900, Dallas, Texas 75221. 
value problem. Like Baker's scheme, this scheme is analogous to $H^{-1}$-Galerkin methods, more specifically to the collocation- $H^{-1}$-Galerkin methods proposed by Díaz [5] and Dunn and Wheeler [6].

In Section 2, the collocation-Galerkin scheme is defined and some notation is introduced. In Section 3, the convergence analysis is presented. To this end, we introduce a first order projection with respect to the space variable and derive optimal global estimates for this projection. The remainder of the analysis consists of comparing the collocation-Galerkin solution with the first order projection. The use of estimates derived for this projection and the triangle inequality then leads to the desired result. Similar ideas have been used by Díaz [5] to analyze a collocation- $H^{-1}$-Galerkin method for parabolic problems with time-dependent coefficients.

2. The Hyperbolic Problem and Some Notation. In this section, we describe the first order initial-boundary value problem under consideration, introduce the continuous in time collocation-Galerkin scheme for this type of problem, and define some notation to be used in the analysis of the scheme.

2.1. The Initial-Boundary Value Problem. Consider the first order hyperbolic initial-boundary value problem

$$
\begin{aligned}
\frac{\partial u}{\partial t}+\frac{\partial}{\partial x}(a(x, t) u) & =f(x, t), \quad(x, t) \in I \times J, \\
u(0, t) & =g(t), \quad t \in J, \\
u(x, 0) & =u_{0}(x), \quad x \in I,
\end{aligned}
$$

where $I=(0,1)$ and $J=(0, T)$. Assume $a, a_{x}, a_{x x}, a_{t}, a_{t x}$ and $a_{t x x} \in L^{\infty}(I)$, uniformly in $t$. Also assume that there exists a positive constant $a_{0}$ such that

$$
a_{0}<a(x, t), \quad(x, t) \in I \times \bar{J} .
$$

Before describing the collocation-Galerkin approximation to (2.1), we introduce some notation. Let $\delta=\left\{0=x_{0}<x_{1}<\cdots<x_{N}=1\right\}$ be a partition of $I$ with $I_{i}=\left(x_{i-1}, x_{i}\right), h_{i}=x_{i}-x_{i-1}$, for $i=1, \ldots, N$ and $h=\max _{1<i<N} h_{i}$. We assume that the class of partitions $\delta$ is quasiuniform; that is, there is a constant $\sigma$, independent of $h$, such that $\sigma h \leqslant \min _{1<i<N} h_{i}$. For a positive integer $r$, denote by $P_{r}(D)$ the class of polynomials of degree not greater than $r$ restricted to the set $D$, and let

$$
\mathfrak{N}_{-1}^{r} \equiv \mathfrak{N C}_{-1}^{r}(\delta)=\left\{v:\left.v\right|_{I_{i}} \in P_{r}\left(I_{i}\right), 1 \leqslant i \leqslant N\right\},
$$

and for an integer $k$ satisfying $0 \leqslant k \leqslant r$, define

$$
\mathfrak{T K}_{k}^{r} \equiv \mathfrak{N}_{k}^{r}(\delta)=H^{k+1}(I) \cap \mathfrak{M K}_{-1}^{r} \cap\{v: v(1)=0\}
$$

Let $J_{r-1}$ be the Jacobi polynomial of degree $r-1$ on $I$ with respect to the weight function $x(1-x)$. The collocation points are defined to be the affine images in each subinterval of the roots $\rho_{j}$ of $J_{r-1}$. The $N(r-1)$ collocation points are then given by

$$
x_{i j}=x_{i-1}+h_{i} \rho_{j}, \quad 1 \leqslant j \leqslant r-1,1 \leqslant i \leqslant N .
$$

The collocation-Galerkin method determines a differentiable map $U:[0, T] \rightarrow$ $\mathfrak{T}_{-1}^{r-1}$ which satisfies $(2.1)$ at the $N(r-1)$ collocation points and a weak-weak 
form of (2.1) with respect to $\mathfrak{R}_{0}^{1}$. Thus, for $t \in(0, T]$, we require

$$
\begin{aligned}
& \left(U_{t}+(a U)_{x}-f\right)\left(x_{i j}, t\right)=0, \quad 1 \leqslant j \leqslant r-1,1<i<N, \\
& \left(U_{t}, V\right)-\left(U, a V_{x}\right)=(f, V)+a(0, t) g(t) V(0), \quad V \in \Re_{0}^{1},
\end{aligned}
$$

and for $t=0$

$$
\begin{gathered}
\left.\frac{\partial}{\partial x}(a(U-u))\right|_{x=x_{i j}}=0, \quad 1 \leqslant j \leqslant r-1,1 \leqslant i<N, \\
\left(U-u_{0}, a V_{x}\right)=0, \quad V \in \mathbb{R}_{0}^{1},
\end{gathered}
$$

where $(\phi, \psi)=\int_{I} \phi, \psi$ is the $L^{2}$-innerproduct on $I$.

2.2. Semidiscrete Innerproducts. In order to analyze the method (2.2), we restate it in a semidiscrete variational form that uses the semidiscrete innerproduct and bilinear forms that are introduced in this section.

A subspace of $\Re_{0}^{r}$ important to our presentation is defined by

$$
Z_{0}^{r} \equiv Z_{0}^{r}(\delta)=\left\{v \in \mathfrak{T}_{0}^{r} \mid v\left(x_{i}\right)=0, i=0,1, \ldots, N\right\} .
$$

$Z_{0}^{r}$ can be characterized by the property

$$
\mathfrak{M}_{0}^{r}=Z_{0}^{r} \oplus \mathfrak{M}_{0}^{1} .
$$

Let the positive weights $w_{i}, i=1, \ldots, r-1$, be the unique choices such that

$$
\int_{0}^{1} x(1-x) p(x) d x=\sum_{j=1}^{r-1} w_{j} p\left(\rho_{j}\right), \quad p \in P_{2 r-3}(I) .
$$

Let $v$ and $\phi$ be defined on $I$, and let $v$ satisfy $v\left(x_{i}\right)=0,0 \leqslant i \leqslant N$. Then define the discrete bilinear form

$$
\langle\phi, v\rangle_{i}=h_{i} \sum_{j=1}^{r-1} w_{j} \frac{\phi\left(x_{i j}\right) v\left(x_{i j}\right)}{\rho_{j}\left(1-\rho_{j}\right)}, \quad 1 \leqslant i<N,
$$

and

$$
\langle\phi, v\rangle=\sum_{i=1}^{N}\langle\phi, v\rangle_{i} .
$$

Note that if $\phi \in P_{r-1}\left(I_{i}\right)$ and $v \in Z_{0}^{r}$,

$$
(\phi, v)_{i} \equiv \int_{I_{i}} \phi(x) v(x) d x=\langle\phi, v\rangle_{i}
$$

Now let $\psi$ be defined on $I$ and let $\psi_{i} \in \Re_{0}^{1}$ satisfy $\left(\psi-\psi_{1}\right)\left(x_{i}\right)=0,0 \leqslant i \leqslant N$. Let $\psi_{2}=\psi-\psi_{1}$, and note that $\psi_{2}\left(x_{i}\right)=0,0<i \leqslant N$. For a function $\phi$ defined on $I$, let

$$
\langle\phi, \psi\rangle_{i}=\left\langle\phi, \psi_{2}\right\rangle_{i}+\left(\phi, \psi_{1}\right)_{i}, \quad 1 \leqslant i \leqslant N
$$

Notice that if $\phi \cdot \psi \in P_{2 r-1}\left(I_{i}\right)$, then

$$
\langle\phi, \psi\rangle_{i}=(\phi, \psi)_{i} .
$$

In particular, if $\phi \in \mathfrak{N}_{-1}^{r-1}$ and $\psi \in \mathfrak{N}_{0}^{r}$, then

$$
\langle\phi, \psi\rangle=(\phi, \psi) \text {. }
$$


For a function $a \in L^{2}\left(I_{i}\right)$ with first derivative $a^{\prime}$ defined on each $I_{i}$, we define an additional semidiscrete bilinear form $\mathcal{H}(\cdot, \cdot)$ by

$$
\mathcal{H}(\phi, \psi)=\left\langle\frac{d}{d x}(a \phi), \psi_{2}\right\rangle-\left(\phi, a \frac{d \psi_{1}}{d x}\right),
$$

where $\psi_{1}$ and $\psi_{2}$ are as above. Note that if $\phi \in \mathfrak{N}_{-1}^{r}$ and $\psi \in \mathfrak{T}_{0}^{r}$, then

$$
\mathcal{H}(\phi, \psi)=-\left(\phi, a \frac{d}{d x} \psi\right)
$$

when $a \in P_{1}\left(I_{i}\right)$, for each $I_{i}$.

With the foregoing notation, it follows that $U(\cdot, t) \in \mathfrak{N}_{-1}^{r-1}$ satisfying (2.2) also satisfies

$$
\left\langle U_{t}, V\right\rangle+\mathcal{H}(U, V)=\langle f, V\rangle+a(0, t) g(t) V(0), \quad V \in \mathfrak{N}_{0}^{r}
$$

The use of relations (2.5) on Eqs. (2.6) shows that the collocation-Galerkin method is equivalent to finding $U:[0, T] \rightarrow \mathfrak{N}_{-1}^{r-1}$ satisfying

$$
\begin{gathered}
\left(U_{t}, V\right)+\mathcal{H}(U, V)=\langle f, V\rangle+a(0, t) g(t) V(0), \quad V \in \mathcal{M}_{0}^{r}, t \in J, \\
\mathcal{H}\left(U(\cdot, 0)-u_{0}, V\right)=0, \quad V \in \mathscr{T}_{0}^{r} .
\end{gathered}
$$

Notice that because the solution $u$ to the initial-boundary value problem (2.1) also satisfies Eqs. (2.6), we have

$$
\left(U_{t}, V\right)+\mathcal{H}(U, V)=\left\langle u_{t}, V\right\rangle+\mathcal{H}(u, V), \quad V \in \mathfrak{N}_{0}^{r}, t \in J
$$

Before proceeding with the analysis, we introduce some additional notation. Let $E$ denote a fixed interval. For integer $s, H^{s}(E)$ denotes the closure of $C^{\infty}(E)$ in the norm

$$
\|f\|_{H^{s}(E)}=\left(\sum_{i=0}^{s}\left\|f^{(i)}\right\|_{L^{2}(E)}^{2}\right)^{1 / 2}, \quad s \geqslant 0
$$

and

$$
\|f\|_{H^{s}(E)}=\sup _{0 \neq \chi \in C^{\infty}(E)} \frac{\left|\int_{E} f(\xi) \chi(\xi) d \xi\right|}{\|\chi\|_{H^{-s}(E)}}, \quad s<0,
$$

respectively, where $\|f\|_{L^{2}(E)}^{2}=\int_{E} f^{2}(\xi) d \xi$. Also, let $T \in(0, \infty)$ be fixed, then define for $p=2, \infty$

$$
L^{p}\left(0, T ; H^{s}(E)\right)=\left\{v:[0, T] \rightarrow H^{s}(E) \mid\|v\|_{L^{p}\left(H^{s}(E)\right)} \leqslant \infty\right\},
$$

where

$$
\|v\|_{L^{2}\left(H^{s}(E)\right)}^{2}=\left(\int_{0}^{T}\|v(\cdot, t)\|_{H^{s}(E)}^{2} d t\right)^{1 / 2}
$$

and

$$
\|v\|_{L^{\infty}\left(H^{s}(E)\right)}=\sup _{0<t<T}\|v(\cdot, t)\|_{H^{s}(E)} .
$$

Also for $q$ a positive integer, let

$$
\|v\|_{H^{q}\left(0, T ; H^{s}(E)\right)}=\left(\sum_{k=0}^{q} \int_{0}^{T}\left\|\frac{\partial^{k} v}{\partial t^{k}}\right\|_{H^{s}(E)}^{2} d t\right)^{1 / 2} .
$$

For simplicity we shall suppress the dependence of $E$ whenever $E=I$. 
An estimate for the $H^{-1}$-norm of elements of $\mathfrak{N}_{-1}^{r-1}$, which we use in our analysis, is given in the following lemma. The proof is presented in the appendix.

LEMMA 2.1. If $F \in \mathfrak{T}_{-1}^{r-1}$, then there is a constant $C$, independent of $h$, such that

$$
\|F\|_{H^{-1}} \leqslant|(F, 1)|+C \sup _{0 \neq \chi \in \mathscr{N}_{0}^{r}} \frac{|(F, \chi)|}{\|\chi\|_{L^{2}}} .
$$

We now state some well-known inequalities that we shall use later. Let $\mu$ be the length of the interval $E$. There exist constants $C_{1}$ and $C_{2}$, independent of $\mu$, such that for $\phi \in H^{1}(E)$

$$
\|\phi\|_{L^{\infty}(E)} \leqslant C_{1}\left\{\mu^{1 / 2}\left\|\phi^{\prime}\right\|_{L^{2}(E)}+\mu^{-1 / 2}\|\phi\|_{L^{2}(E)}\right\}
$$

and for $\phi \in P_{s}(E), s>0$,

$$
\left\|\phi^{\prime}\right\|_{L^{2}(E)} \leqslant C_{2} \mu^{-1}\|\phi\|_{L^{2}(E)} \text { (inverse property). }
$$

3. Error Analysis. Let $u$ denote the solution to (2.1), and let the map $U$ : $[0, T] \rightarrow \Re_{-1}^{r}$ denote the solution to (2.3).

The central result of this paper is given by the following theorem.

THEOREM 3.1. Let $u$ denote the solution to (2.1), and let the map $U:[0, T] \rightarrow \mathfrak{M}_{-1}^{r-1}$ be the solution to (2.2). If $u, u_{t}$, and $u_{t t} \in L^{2}\left(0, t: H^{r}(I)\right)$, then for $h$ sufficiently small, there exists a constant $C$ independent of $h$, such that

$$
\begin{aligned}
\|u-U\|_{L^{\infty}\left(0, T ; L^{2}\right)} \leqslant C\left\{\left(\sum_{i=1}^{N} h_{i}^{2 r}\|u\|_{L^{\infty}\left(0, T ; H^{r}\left(I_{i}\right)\right)}^{2}\right)^{1 / 2}\right. & \\
& \left.+h\left(\sum_{i=1}^{N} h_{i}^{2 r-2}\|u\|_{H^{2}\left(0, T ; H^{r-1}\left(I_{i}\right)\right)}^{2}\right)^{1 / 2}\right\} .
\end{aligned}
$$

In order to prove the theorem, we introduce the first order projection. In the following two sections, we compare this projection to both $u$ and $U$, obtaining the estimates that we use in the proof of this theorem.

3.1. First Order Projection. In this section we introduce a map $W:[0, T] \rightarrow \mathfrak{N}_{-1}^{r-1}$ via a first order projection of $u$ with respect to the space variable and derive error estimates for this projection. Since the coefficient $a$ is time-dependent, in this section we also obtain estimates for time derivatives of $u-W$.

The map $W:[0, T] \rightarrow \mathbb{N}_{-1}^{r-1}$ is defined, for $t \in \bar{J}$, by

$$
\frac{\partial}{\partial x}(a W)\left(x_{i j}, t\right)=\frac{\partial}{\partial x}(a u)\left(x_{i j}, t\right), \quad 1 \leqslant j \leqslant r-1,1<i \leqslant N,
$$

and

$$
\left(W, a V_{x}\right)=\left(u, a V_{x}\right), \quad V \in \mathfrak{T}_{0}^{1}
$$

The estimates of $u-W$ are contained in the following theorem which summarizes the results of this section.

THEOREM 3.2. Let $u$ be the solution of the initial-boundary value problem (2.1) and $W$ the solution of Eqs. (3.1). If, for some integer $k \geqslant 0$,

$$
\frac{\partial^{k} u}{\partial t^{k}} \in L^{p}\left(0, T ; H^{r}(I)\right),
$$


then

$$
\frac{\partial^{k} W}{\partial t^{k}} \in L^{p}\left(0, T ; \Re_{-1}^{r-1}\right)
$$

Furthermore, there exists a constant $C$ such that

$$
\left\|\frac{\partial^{k}}{\partial t^{k}}(u-W)\right\|_{L^{p}\left(L^{2}\right)} \leqslant C \sum_{j=0}^{k}\left(\sum_{i=1}^{N} h_{i}^{2 r}\left\|\frac{\partial^{j} u}{\partial t^{j}}\right\|_{L^{p}\left(H^{\prime}\left(I_{i}\right)\right)}^{2}\right)^{1 / 2} .
$$

We divide the proof of Theorem 3.2 into several lemmas. In Lemmas 3.2 and 3.3 we consider the case $k=0$. The case $k \geqslant 1$ is a consequence of Lemma 3.4. In Lemma 3.1 we derive a duality result that is used in the analysis of the first order projection.

LEMMA 3.1. Let $\eta \in L^{2}(I)$ with $\eta_{x} \in L^{2}\left(I_{i}\right), 1 \leqslant i \leqslant N$. Let $\xi \in H^{1}(I)$ satisfy $\xi(1)=0$ and

$$
-a \frac{\partial \xi}{\partial x}=\eta, \quad x \in I .
$$

Also let $\chi \in \mathfrak{T}_{0}^{1}$ satisfy $\chi\left(x_{i}\right)=\xi\left(x_{i}\right), 1<i \leqslant N$. Then for $h$ sufficiently small there exists a constant $C$, independent of $h$, such that

$$
\|\eta\|_{L^{2}}^{2} \leqslant C\left\{\sum_{i=1}^{N} h_{i}\left\|\eta_{x}\right\|_{L^{2}\left(I_{i}\right)}\left\|\xi_{x}\right\|_{L^{2}\left(I_{i}\right)}\right\}-\left(\eta, a \frac{\partial}{\partial x} \chi\right) .
$$

Proof. By the assumptions on $a$ it follows that

Let $\zeta=\xi-\chi ;$ then

$$
\left\|\frac{\partial \xi}{\partial x}\right\|_{L^{2}} \leqslant C\|\eta\|_{L^{2}}
$$

$$
(\eta, \eta)=-\left(\eta, a \frac{\partial \xi}{\partial x}\right)=-\left(\eta, a \frac{\partial \zeta}{\partial x}\right)-\left(\eta, a \frac{\partial \chi}{\partial x}\right)
$$

Notice that $\zeta\left(x_{i}\right)=0$; thus

$$
\begin{aligned}
-\left(\eta, a \frac{\partial \zeta}{\partial x}\right) & =\left(\eta, \zeta \frac{\partial a}{\partial x}\right)+\sum_{i=1}^{N}\left(\frac{\partial \eta}{\partial x}, a \zeta\right)_{i} \\
& \leqslant C\left\{\|\eta\|_{L^{2}}\|\zeta\|_{L^{2}}+\sum_{i=1}^{N}\left\|\eta_{x}\right\|_{L^{2}\left(I_{i}\right)}\|\zeta\|_{L^{2}\left(I_{i}\right)}\right\} \\
& \leqslant C\left\{h\|\eta\|_{L^{2}}^{2}+\sum_{i=1}^{N} h_{i}\left\|\eta_{x}\right\|_{L^{2}\left(I_{i}\right)}\left\|\xi_{x}\right\|_{L^{2}\left(I_{i}\right)}\right\}
\end{aligned}
$$

Thus taking $h$ sufficiently small concludes the proof of the lemma.

In order to prove Theorem 3.2, we introduce a map $R:[0, T] \rightarrow \mathfrak{T}_{-1}^{r-1}$ and derive error estimates for $u-R$ and $W-R$. The map $R$ satisfies, for $t \in J$,

$$
\frac{\partial R}{\partial x}\left(x_{i j}, t\right)=\frac{\partial u}{\partial x}\left(x_{i j}, t\right), \quad 1 \leqslant j \leqslant r-1,1 \leqslant i \leqslant N,
$$

and

$$
\left(R, a V_{x}\right)=\left(u, a V_{x}\right), \quad V \in \Re_{0}^{1}
$$

Estimates for $u-R$ are presented in the following lemma. 
LEMMA 3.2. Let $u$ be the solution to (2.1) and the map $R$ be the solution to (3.4). If $u \in H^{r}(I)$, then, for $h$ sufficiently small, there exist constants $C_{1}$ and $C$, independent of $h$, such that

$$
\left\|\frac{\partial}{\partial x}(u-R)\right\|_{L^{2}\left(I_{i}\right)} \leqslant C_{1} h_{i}^{r-1}\|u\|_{H^{r}\left(I_{i}\right)}
$$

and

$$
\|u-R\|_{L^{2}} \leqslant C\left(\sum_{i=1}^{N} h_{i}^{2 r}\|u\|_{H^{\prime}\left(I_{i}\right)}^{2}\right)^{1 / 2}
$$

Proof. We first demonstrate the existence and uniqueness of $R$. From (3.4i), by interpolation we conclude the existence and uniqueness of $R_{x}$. To show that $R$ is unique, we assume that both $R_{1}$ and $R$ satisfy (3.4). Then $E=R_{1}-R$ is a constant on each subinterval $I_{i}$ that we call $\beta_{i}$. Now, using the Galerkin equations (3.4ii), we find

$$
\left(E, a V_{x}\right)=0, \quad V \in \mathfrak{M}_{0}^{1} .
$$

For $i=0,1, \ldots, N-1$, let $V_{i}$ be the continuous piecewise-linear function such that $V_{i}\left(x_{j}\right)=\delta_{i j}, 0 \leqslant j \leqslant N$. The space $\Re_{0}^{1}$ is the linear span of these functions. Hence by taking $V=V_{i}, i=0, \ldots, N-1$, since $a(x, t) \geqslant a_{0}>0$ for $(x, t) \in I$ $\times \bar{J}$

$$
\int_{x_{i-1}}^{x_{i}} a d x>0, \quad i=1, \ldots, N
$$

Thus $\beta_{1}=\beta_{2}=\cdots=\beta_{N}=0$, which implies that $E \equiv 0$ and establishes the existence and uniqueness of the solution $R$ to (3.4). By (3.4i) and interpolation at the collocation points, we have

$$
\left\|\frac{\partial}{\partial x}(u-R)\right\|_{L^{2}\left(I_{i}\right)} \leqslant C_{1} h_{i}^{r-1}\|u\|_{H^{r}\left(I_{i}\right)}
$$

Let $\psi=u-R$, and let $\phi \in H^{1}(I)$ satisfy $\phi(1)=0$, and

$$
-a \frac{\partial \phi}{\partial x}=\psi, \quad x \in I
$$

Hence, by Lemma 3.1 and (3.4ii),

$$
\|\psi\|_{L^{2}}^{2} \leqslant C\left(\sum_{i=1}^{N} h_{i}^{2}\left\|\psi_{x}\right\|_{L^{2}\left(I_{i}\right)}^{2}\right)^{1 / 2}
$$

The estimates for $W-R$ are contained in the following lemma.

LEMMA 3.3. Let $u$ and the maps $W$ and $R$ be the solutions to (2.1), (3.1), and (3.4), respectively. Then, for $h$ sufficiently small, there exists a constant $C$, independent of $h$, such that

$$
\|W-R\|_{L^{2}} \leqslant C\left\{h\|u-R\|_{L^{2}}+\left(\sum_{i=1}^{N} h_{i}^{4}\left\|\frac{\partial}{\partial x}(u-R)\right\|_{L^{2}\left(I_{i}\right)}^{2}\right)^{1 / 2}\right\} .
$$


Proof. Let $E=W-R$ and $e=u-R$. Then, at the collocation points because of (3.1i) and (3.4i), we have

$$
\begin{aligned}
\frac{\partial(a E)}{\partial x}\left(x_{i j}\right) & =\frac{\partial}{\partial x}(a(W-u))\left(x_{i j}\right)+\frac{\partial}{\partial x}(a(u-R))\left(x_{i j}\right) \\
& =\frac{\partial}{\partial x}(a e)\left(x_{i j}\right)=a e_{x}\left(x_{i j}\right)+a_{x} e\left(x_{i j}\right)=a_{x} e\left(x_{i j}\right)
\end{aligned}
$$

Since

$$
v_{x}=\frac{(a v)_{x}}{a}-\frac{a_{x} v}{a}
$$

then

$$
E_{x}\left(x_{i j}\right)=\left(-\frac{a_{x}}{a} E+\frac{a_{x}}{a} e\right)\left(x_{i j}\right)
$$

and

$$
\left|E_{x}\left(x_{i j}\right)\right| \leqslant C\left\{\|E\|_{L^{\infty}\left(I_{i}\right)}+\|e\|_{L^{\infty}\left(I_{i}\right)}\right\}
$$

Hence

$$
\left\|E_{x}\right\|_{L^{\infty}\left(I_{i}\right)} \leqslant C\left\{\|E\|_{L^{\infty}\left(I_{i}\right)}+\|e\|_{L^{\infty}\left(I_{i}\right)}\right\} .
$$

Using inequalities (2.9) and (2.10), we have

$$
\begin{aligned}
\left\|E_{x}\right\|_{L^{\infty}\left(I_{i}\right)} & \leqslant C\left\{h_{i}^{-1 / 2}\|E\|_{L^{2}\left(I_{i}\right)}+h_{i}^{1 / 2}\left\|E_{x}\right\|_{L^{2}\left(I_{i}\right)}+h_{i}^{-1 / 2}\|e\|_{L^{2}\left(I_{i}\right)}+h_{i}^{1 / 2}\left\|e_{x}\right\|_{L^{2}\left(I_{i}\right)}\right\} \\
& \leqslant C\left\{h_{i}^{-1 / 2}\|E\|_{L^{2}\left(I_{i}\right)}+h_{i}^{-1 / 2}\|e\|_{L^{2}\left(I_{i}\right)}+h_{i}^{1 / 2}\left\|e_{x}\right\|_{L^{2}\left(I_{i}\right)}\right\} .
\end{aligned}
$$

It then follows that

$$
\left\|E_{x}\right\|_{L^{2}\left(I_{i}\right)} \leqslant C\left\{\|E\|_{L^{2}\left(I_{i}\right)}+\|e\|_{L^{2}\left(I_{i}\right)}+h_{i}\left\|e_{x}\right\|_{L^{2}\left(I_{i}\right)}\right\} .
$$

Now, we use duality. Let $\phi \in H^{1}(I)$ satisfy $\phi(1)=0$, and

$$
a \frac{\partial \phi}{\partial x}=-E, \quad x \in I .
$$

Thus, by Lemma 3.1, (3.1ii) and (3.4ii), we obtain

$$
\begin{aligned}
\|E\|_{L^{2}}^{2} & \leqslant\left(\sum_{i=1}^{N}\left\|E_{x}\right\|_{L^{2}\left(I_{i}\right)} h_{i}\left\|\frac{\partial \phi}{\partial x}\right\|_{L^{2}\left(I_{i}\right)}\right) \\
& \leqslant C\left(h\|E\|_{L^{2}}\left\|\frac{\partial \phi}{\partial x}\right\|_{L^{2}}+h\|e\|_{L^{2}}\left\|\frac{\partial \phi}{\partial x}\right\|_{L^{2}}+\sum_{i=1}^{N} h_{i}^{2}\left\|e_{x}\right\|_{L^{2}\left(I_{i}\right)}\left\|\frac{\partial \phi}{\partial x}\right\|_{L^{2}\left(I_{i}\right)}\right),
\end{aligned}
$$

where we have used (3.5). Hence, using the Cauchy-Schwarz inequality, we obtain

$$
\|E\|_{L^{2}}^{2} \leqslant C\left(h\|E\|_{L^{2}}+h\|e\|_{L^{2}}+\left(\sum_{i=1}^{N} h_{i}^{4}\left\|e_{x}\right\|_{L^{2}\left(I_{i}\right)}^{2}\right)^{1 / 2}\right)\|E\|_{L^{2}}
$$

Thus, on choosing $h$ sufficiently small, we obtain

$$
\|E\|_{L^{2}} \leqslant C\left\{h\|e\|_{L^{2}}+\left(\sum_{i=1}^{N} h_{i}^{4}\left\|e_{x}\right\|_{L^{2}\left(I_{i}\right)}^{2}\right)^{1 / 2}\right\}
$$

which proves the lemma. 
The estimates (3.2) for $k>0$ are derived via an inductive argument. However, in order to avoid cumbersome details, we show the induction step for $W_{t}$. To do this consider a map $Y:[0, t] \rightarrow \Re_{-1}^{r-1}$ satisfying, for $t \in \bar{J}$,

$$
\frac{\partial}{\partial x}(a Y)\left(x_{i j}, t\right)=\frac{\partial}{\partial x}\left(a u_{t}\right)\left(x_{i j}, t\right), \quad 1 \leqslant j \leqslant r-1,1 \leqslant i \leqslant N,
$$

and

$$
\left(Y, a v_{x}\right)=\left(u_{t}, a v_{x}\right), \quad V \in \mathfrak{R}_{0}^{1} .
$$

Optimal estimates for $Y-u_{t}$ follow from Lemmas 3.2 and 3.3. We compare $W_{t}$ and $Y$ in the following lemma.

LEMMA 3.4. Let $u$ be the solution to (2.1), the map $W$ be the solution to (3.1), and the map $Y$ the solution to (3.6). Then, for $h$ sufficiently small, there exists a constant $C$, independent of $h$, such that

$$
\left\|Y-W_{t}\right\|_{L^{2}} \leqslant C\|u-W\|_{L^{2}}+C\left\{\sum_{i=1}^{N} h_{i}^{4}\left\|(u-W)_{x}\right\|_{L^{2}\left(I_{i}\right)}^{2}\right\}^{1 / 2} .
$$

Proof. Let $\eta=W_{t}-Y$ and $e=u-W$. Then, from (3.1) and (3.6),

$$
\begin{aligned}
\frac{\partial}{\partial x}(a \eta)\left(x_{i j}, t\right) & =\left[-\frac{\partial}{\partial x}\left(a e_{t}\right)+\frac{\partial}{\partial x}\left(a\left(u_{t}-Y\right)\right)\right]\left(x_{i j}, t\right) \\
& =\left[-\frac{\partial^{2} a e}{\partial t \partial x}+\frac{\partial}{\partial x}\left(a_{t} e\right)\right]\left(x_{i j}, t\right)=\frac{\partial}{\partial x}\left(a_{t} e\right)\left(x_{i j}, t\right) \\
& =\left[a_{t x} e+a_{t} e_{x}\right]\left(x_{i j}, t\right) .
\end{aligned}
$$

Note that for sufficiently smooth $\phi$

$$
\phi_{x}=\frac{(a \phi)_{x}}{a}-\frac{a_{x} \phi}{a}
$$

hence

$$
\begin{aligned}
\frac{\partial \eta}{\partial x}\left(x_{i j}, t\right) & =\frac{1}{a}\left[(a \eta)_{x}-a_{x} \eta\right]\left(x_{i j}, t\right)=\frac{1}{a}\left[a_{t x} e-a_{x} \eta+a_{t} e_{x}\right]\left(x_{i j}, t\right) \\
& =\left[e \frac{\partial}{\partial x}\left(\frac{a_{t}}{a}\right)-\eta \frac{a_{x}}{a}\right]\left(x_{i j}, t\right) .
\end{aligned}
$$

Thus, by estimating and using (2.9) and (2.10), we obtain

$$
\left\|\eta_{x}\right\|_{L^{2}\left(I_{i}\right)} \leqslant\left\{\|\eta\|_{L^{2}\left(I_{i}\right)}+\|e\|_{L^{2}\left(I_{i}\right)}+h_{i}\left\|e_{x}\right\|_{L^{2}\left(I_{i}\right)}\right\} .
$$

We use duality now. Let $\phi \in H^{1}$ satisfy $\phi(1)=0$ and

$$
-a \frac{\partial \phi}{\partial x}=\eta, \quad x \in I .
$$

Thus, by Eqs. (3.1ii) and (3.6ii), we have for $\chi \in \mathfrak{T}_{0}^{1}$

$$
\begin{aligned}
-\left(\eta, a \frac{\partial \chi}{\partial x}\right) & =-\left(e, a \frac{\partial \chi}{\partial x}\right) \\
& =-\frac{d}{d t}\left(e, a \frac{\partial \chi}{\partial x}\right)+\left(e, a_{t} \frac{\partial \chi}{\partial x}\right)+\left(e, a \frac{\partial \chi_{t}}{\partial x}\right)=\left(e, a_{t} \frac{\partial \chi}{\partial x}\right) .
\end{aligned}
$$


Hence, if we pick $\chi$ such that $\chi\left(x_{i}\right)=\phi\left(x_{i}\right)$, we obtain by Lemma 3.1, (3.7) and (3.8) that

$$
\begin{aligned}
\|\eta\|_{L^{2}}^{2} & \leqslant\left(e, a_{t} \frac{\partial \chi}{\partial x}\right)+C \sum_{i=1}^{N} h_{i}\left\|\eta_{x}\right\|_{L^{2}\left(I_{i}\right)}\left\|\phi_{x}\right\|_{L^{2}\left(I_{i}\right)} \\
& \leqslant C\left\{\|e\|_{L^{2}}+h\|\eta\|_{L^{2}}+\left(\sum_{i=1}^{N} h_{i}^{2}\left\|\eta_{x}\right\|_{L^{2}\left(I_{i}\right)}^{2}\right)^{1 / 2}\right\}\left\|\phi_{x}\right\|_{L^{2} .}
\end{aligned}
$$

Therefore

$$
\|\eta\|_{L^{2}} \leqslant C\left\{\|e\|_{L^{2}}+h\|\eta\|_{L^{2}}+\left(\sum_{i=1}^{N} h_{i}^{2}\left\|\eta_{x}\right\|_{L^{2}\left(I_{i}\right)}^{2}\right)^{1 / 2}\right\} .
$$

Taking $h$ sufficiently small concludes the proof of the lemma.

The proof of Theorem 3.2 now follows from Lemmas 3.2, 3.3, and 3.4 and the triangle inequality.

Using arguments similar to those used to prove Theorem 3.2, we can derive $H^{-1}$-norm estimates of $u-W$ which will be used in obtaining the final estimates. These estimates are contained in the following corollary to Theorem 3.2.

Corollary 3.1. Let $u$ and $W$ be as in Theorem 3.2. Then there is a constant $C$, independent of $h$, such that

$$
\left\|\frac{\partial^{k}}{\partial t^{k}}(u-W)\right\|_{H^{-1}} \leqslant C h \sum_{j=0}^{k}\left\|\frac{\partial^{j}}{\partial t^{j}}(u-W)\right\|_{L^{2}} .
$$

Proof. The case $k=0$ is proved similar to Theorem 3.2 of Baker [2]. For $k>1$, use Eq. (3.8) and proceed similarly to the arguments in Lemma 3.4.

3.2. Estimation of $U-W$. In this section we compare the maps $U$ and $W$. To do this, we derive some quadrature estimates due to the semidiscrete innerproduct and use them to obtain estimates for $E=U-W$. There are two basic estimates that we derive. First we estimate $\left\|E_{t}\right\|_{H^{-1}}$. Then $\|E\|_{L^{2}}$ is estimated. The estimates for $E$ are contained in the following theorem.

THEOREM 3.3. Let $u$ be the solution to (2.1) and let the maps $U$ and $W$ : $[0, T] \rightarrow \mathfrak{N}_{-1}^{r-1}$ be the solutions to (2.2) and (3.1), respectively. If $u, u_{t}$, and $u_{t t} \in$ $L^{2}\left(0, T ; H^{2}(I)\right)$, then, for $h$ sufficiently small, there is a constant $C$, independent of $h$, such that for $1 \leqslant s \leqslant r-1$

$$
\|U-W\|_{L^{\infty}\left(L^{2}\right)}^{2} \leqslant C h^{2}\left\{\|u-W\|_{H^{2}\left(L^{2}\right)}^{2}+\sum_{i=1}^{N} h_{i}^{2 s}\left\|\frac{\partial u}{\partial t}\right\|_{H^{1}\left(H^{s}\left(I_{i}\right)\right)}^{2}\right\} \text {. }
$$

In order to prove Theorem 3.3 , we first derive some basic results. Let $\varepsilon=$ $u-W$. From (2.8) and (3.1) we obtain

$$
\left(\frac{\partial}{\partial t}(U-W), V\right)+\mathcal{H}(U-W, V)=\left\langle\varepsilon_{t}, V\right\rangle, \quad V \in \mathfrak{N} \boldsymbol{r}_{0}
$$

For $\phi \in L^{2}$ and defined on each $I_{i}$ and $V \in \mathfrak{T}_{0}^{r}$, let

$$
q_{i}(\phi, V)=\langle\phi, V\rangle_{i}-(\phi, V)_{i} \text { and } q(\phi, V)=\sum_{i=1}^{N} q_{i}(\phi, V) \text {. }
$$


Also, for $R \in \mathfrak{N}_{-1}^{r-1}$ and $V \in \mathfrak{N}_{0}^{r}$, let

$$
Q(a, R, V)=-\left(R, a \frac{\partial V}{\partial x}\right)+\mathcal{H}(R, V) .
$$

Hence, we can rewrite (3.9) as

$$
\left(E_{t}, V\right)-\left(E, a \frac{\partial V}{\partial x}\right)=\left(\varepsilon_{t}, V\right)+q\left(u_{t}, V\right)+Q(a, E, V), \quad V \in \mathfrak{N r}
$$

In order to derive the error estimates, we must estimate the quadrature errors $q$ and $Q$. These estimates are a consequence of the following two lemmas.

LEMMA 3.5. Let $\phi \in H^{s}\left(I_{i}\right), 1 \leqslant s \leqslant r$, and $V \in \mathfrak{M}_{0}^{r}$. Then, there exists a constant $C$, independent of $h$, such that

$$
\left|q_{i}(\phi, V)\right| \leqslant C h_{i}^{s+1}\|\phi\|_{H^{s}\left(I_{i}\right)}\left\|V^{\prime}\right\|_{L^{2}\left(I_{i}\right)}
$$

Proof. Because of (2.3), for $V \in \mathfrak{N}_{0}^{r}$ there are unique $V_{1} \in \mathfrak{N}_{0}^{1}$ and $V_{2} \in Z_{0}^{r}$, such that $V=V_{1}+V_{2}$. Moreover,

$$
\left\|V_{2}^{\prime}\right\|_{L^{2}\left(I_{i}\right)}=\left\|\left(V-V_{1}\right)^{\prime}\right\|_{L^{2}\left(I_{i}\right)}<C\left\|V^{\prime}\right\|_{L^{2}\left(I_{i}\right)}
$$

and $q_{i}(\phi, V)=q_{i}\left(\phi, V_{2}\right)$. Choose $\chi \in P_{s-1}\left(I_{i}\right)$ such that

$$
\|\phi-\chi\|_{L^{2}\left(I_{i}\right)}+h_{i}\left\|(\phi-\chi)^{\prime}\right\|_{L^{2}\left(I_{i}\right)}<C h_{i}^{s}\|\phi\|_{H^{s}\left(I_{i}\right)} .
$$

Note that, for $V \in \mathscr{T} r_{0}^{r}$ and $1 \leqslant s \leqslant r, q_{i}(\chi, V)=0$. Let $\psi=\phi-\chi$. Then

$$
\begin{aligned}
q_{i}\left(\phi, V_{2}\right) & =q_{i}\left(\chi, V_{2}\right)+q_{i}\left(\psi, V_{2}\right)=q_{i}\left(\psi, V_{2}\right) \\
& =h_{i} \sum_{j=1}^{N} w_{j} \frac{\psi\left(x_{i j}\right) V_{2}\left(x_{i j}\right)}{\rho_{j}\left(1-\rho_{j}\right)}-\int_{x_{i}}^{x_{i+1}} \psi(\xi) V_{2}(\xi) d \xi \\
& \leqslant C h_{i}\|\psi\|_{L^{\infty}\left(I_{i}\right)}\left\|V_{2}\right\|_{L^{\infty}\left(I_{i}\right)}+C\|\psi\|_{L^{2}\left(I_{i}\right)}\left\|V_{2}\right\|_{L^{2}\left(I_{i}\right)} \\
& \leqslant C h_{i}\left\{\|\psi\|_{L^{2}\left(I_{i}\right)}+h_{i}\left\|\psi^{\prime}\right\|_{L^{2}\left(I_{i}\right)}\right\}\left\|V_{2}^{\prime}\right\|_{L^{2}\left(I_{i}\right)}+C\|\psi\|_{L^{2}\left(I_{i}\right)} h_{i}\left\|V_{2}^{\prime}\right\|_{L^{2}\left(I_{i}\right)} \\
& \leqslant C h_{i}^{s+1}\|\phi\|_{H^{s}\left(I_{i}\right)}\left\|V^{\prime}\right\|_{L^{2}\left(I_{i}\right)},
\end{aligned}
$$

where we have used inequalities (2.9), (2.10), and (3.11). This completes the proof of the lemma.

LemMA 3.6. Let $a^{\prime \prime} \in L^{\infty}\left(I_{i}\right), R \in \mathfrak{T}_{-1}^{r-1}$, and $V \in \mathfrak{T}_{0}^{r}$. Then there exists a constant $C$, independent of $h$, such that

$$
\left|q_{i}\left(\frac{d}{d x}(a R), V\right)\right| \leqslant C h_{i}^{2}\left\|a^{\prime \prime}\right\|_{L^{\infty}\left(I_{i}\right)}\|R\|_{L^{2}\left(I_{i}\right)}\left\|V^{\prime}\right\|_{L^{2}\left(I_{i}\right)}
$$

Proof. As in the previous lemma, it suffices to consider $V \in Z_{0}^{r}$. In this lemma a more careful argument is needed to exploit the fact that $R$ is a piecewise polynomial. Let $\bar{a}_{i} \in P_{1}\left(I_{i}\right)$ such that $a(x)=\bar{a}_{i}(x)+\alpha(x)$, where

$$
\|\alpha\|_{L^{\infty}\left(I_{i}\right)}+h_{i}\left\|\alpha^{\prime}\right\|_{L^{\infty}\left(I_{i}\right)} \leqslant C h_{i}^{2}\left\|a^{\prime \prime}\right\|_{L^{\infty}\left(I_{i}\right)} .
$$

Because of (2.4), we have

$$
q_{i}\left(\frac{d}{d x}(a R), V\right)=q_{i}\left(\frac{d}{d x}(\alpha R), V\right) .
$$


Moreover, since $V \in Z_{0}^{r}$,

$$
q_{i}\left(\frac{d}{d x}(a R), V\right)=\left\langle\frac{d}{d x}(\alpha R), V\right\rangle_{i}+\left(R, \alpha \frac{d V}{d x}\right)_{i}
$$

Now we estimate each term on the right-hand side of (3.12) as follows. For the first term

$$
\begin{aligned}
\left\langle\frac{d}{d x}(\alpha R), V\right\rangle_{i} & =h_{i} \sum_{j=1}^{N} w_{j} \frac{d(\alpha R) /\left.d x\right|_{x=x_{i j}} \int_{x_{i}}^{x_{i j}} V^{\prime}(\xi) d \xi}{\left(\rho_{j}\right)\left(1-\rho_{j}\right)} \\
& \leqslant C h_{i}^{5 / 2}\left\{\|R\|_{L^{\infty}\left(I_{i}\right)}+h_{i}\left\|R^{\prime}\right\|_{L^{\infty}\left(I_{i}\right)}\right\}\left\|a^{\prime \prime}\right\|_{L^{\infty}\left(I_{i}\right)}\left\|V^{\prime}\right\|_{L^{2}\left(I_{i}\right)} \\
& \leqslant C h_{i}^{2}\left\|a^{\prime \prime}\right\|_{L^{\infty}\left(I_{i}\right)}\|R\|_{L^{2}\left(I_{i}\right)}\left\|V^{\prime}\right\|_{L^{2}\left(I_{i}\right)} .
\end{aligned}
$$

In the foregoing we have used inequalities (2.9) and (2.10). The second term is estimated using Cauchy-Schwarz inequality

$$
\begin{aligned}
\left(R, \alpha \frac{d}{d x} V\right)_{i} & \leqslant C\|\alpha\|_{L^{\infty}\left(I_{i}\right)}\|R\|_{L^{2}\left(I_{i}\right)}\left\|V^{\prime}\right\|_{L^{2}\left(I_{i}\right)} \\
& \leqslant C h_{i}^{2}\left\|a^{\prime \prime}\right\|_{L^{\infty}\left(I_{i}\right)}\|R\|_{L^{2}\left(I_{i}\right)}\left\|V^{\prime}\right\|_{L^{2}\left(I_{i}\right)} .
\end{aligned}
$$

Estimates (3.13) and (3.14) and Eq. (3.12) complete the proof of the lemma.

Now, we estimate $E_{t}$ in the $H^{-1}$-norm. For this, we write (3.10) as

$$
\left(E_{t}, V\right)=\left(E, a \frac{\partial V}{\partial x}\right)+\left(\varepsilon_{t}, V\right)+q\left(u_{t}, V\right)+Q(a, E, V), \quad V \in \mathfrak{N}_{0}^{r}
$$

and estimate each term of the right-hand side. The first two terms are estimated as follows:

$$
\left(E, a \frac{\partial V}{\partial x}\right) \leqslant C\|E\|_{L^{2}\|V\|_{H^{1}}} \leqslant C h^{-1}\|E\|_{L^{2}\|V\|_{L^{2}}}
$$

and

$$
\left(\varepsilon_{t}, V\right) \leqslant C\left\|\varepsilon_{t}\right\|_{L^{2}}\|V\|_{L^{2}}
$$

In order to estimate the quadrature terms, we make use of Lemmas 3.5 and 3.6 as follows; by Lemma 3.5, we have, for $1 \leqslant s \leqslant r-1$,

$$
\begin{aligned}
\left|q\left(u_{t}, V\right)\right| & =\left|\sum_{i=1}^{N} q_{i}\left(u_{t}, V\right)\right| \leqslant \sum_{i=1}^{N}\left|q_{i}\left(u_{t}, V\right)\right| \\
& \leqslant C \sum_{i=1}^{N} h_{i}^{s+1}\left\|u_{t}\right\|_{H^{s}\left(I_{i}\right)}\left\|V^{\prime}\right\|_{L^{2}\left(I_{i}\right)} \leqslant C\left(\sum_{i=1}^{N} h_{i}^{2 s}\left\|u_{t}\right\|_{H^{s}\left(I_{i}\right)}^{2}\right)^{1 / 2}\|V\|_{L^{2} .}
\end{aligned}
$$

By Lemma 3.6, we obtain

Hence

$$
|Q(a, E, V)| \leqslant C h\|E\|_{L^{2}\|V\|_{L^{2}}}
$$

$$
\left(E_{t}, V\right) \leqslant C\left(h^{-1}\|E\|_{L^{2}}+\left\|\varepsilon_{t}\right\|_{L^{2}}+\left(\sum_{i=1}^{N} h_{i}^{2 s}\left\|u_{t}\right\|_{H^{s}\left(I_{i}\right)}^{2}\right)^{1 / 2}\right)\|V\|_{L^{2}}
$$

Now it follows by Lemma 2.1 that

$$
\left\|E_{t}\right\|_{H^{-1}} \leqslant C\left(h^{-1}\|E\|_{L^{2}}+\left\|\varepsilon_{t}\right\|_{L^{2}}+\left(\sum_{i=1}^{N} h_{i}^{2 s}\left\|u_{t}\right\|_{H^{s}\left(I_{i}\right)}\right)^{1 / 2}+\left|\left(E_{t}, 1\right)\right|\right)
$$


We now complete the proof of Theorem 3.3 by deriving an estimate for $\|E\|_{L^{2}}$ in terms of $\left\|E_{t}\right\|_{H^{-1}}$.

Let the map $\psi:[0, T] \rightarrow \Re_{0}^{r}$ satisfy

$$
\frac{\partial \psi}{\partial x}(x, t)=-E(x, t), \quad t \in \bar{J}, x \in I .
$$

Then

$$
\|\psi\|_{H^{s}} \leqslant C\|E\|_{H^{s-1}}, \quad s=0,1 .
$$

Choose the map $V:[0, T] \rightarrow \mathfrak{T}_{0}^{r}$ such that

$$
V(x, t)=\frac{\partial \psi}{\partial t}(x, t), \quad t \in \bar{J} ; x \in I .
$$

With this choice of $V$ in (3.10), the terms on the left-hand side of (3.10) can be estimated as follows:

$$
\left(E_{t}, V\right)=-\left(V_{x}, V\right)=\frac{1}{2}(V(0, t))^{2}=\frac{1}{2}\left|\left(E_{t}, 1\right)\right|^{2}
$$

and

$$
\begin{aligned}
-\left(E, a \frac{\partial V}{\partial x}\right) & =\left(E, a E_{t}\right)=\frac{1}{2}\left(\frac{\partial}{\partial t}\left(E^{2}\right), a\right)=\frac{1}{2} \frac{d}{d t}\left(E^{2}, a\right)-\frac{1}{2}\left(E^{2}, a_{t}\right) \\
& \geqslant \frac{1}{2} \frac{d}{d t}\|\sqrt{a} E\|_{L^{2}}^{2}-C\|E\|_{L^{2}}^{2} .
\end{aligned}
$$

Hence, on using (3.18) and (3.19) on (3.10), we obtain for this choice of $V$ that (3.20) $\frac{1}{2} V^{2}(0, t)+\frac{1}{2} \frac{d}{d t}\|\sqrt{a} E\|_{L^{2}}^{2} \leqslant C\|E\|_{L^{2}}^{2}+\left(\varepsilon_{t}, \psi_{t}\right)+q\left(u_{t}, \psi_{t}\right)+Q\left(a, E, \psi_{t}\right)$.

The last term of (3.20) is estimated using Lemma 3.6, (2.10), Cauchy-Schwarz inequality and then estimates (3.17) and (3.16) to obtain

$$
\begin{aligned}
\left|Q\left(a, E, \psi_{t}\right)\right| & \leqslant C h\|E\|_{L^{2}\left\|\psi_{t}\right\|_{L^{2}}} \leqslant C h\|E\|_{L^{2}}\left\|E_{t}\right\|_{H^{-1}} \\
& \leqslant C h\|E\|_{L^{2}}\left\{h^{-1}\|E\|_{L^{2}}+\left(\sum_{i=1}^{N} h_{i}^{2 s}\left\|u_{t}\right\|_{H^{s}\left(I_{i}\right)}^{2}\right)^{1 / 2}+\left|\left(E_{t}, 1\right)\right|+\left\|\varepsilon_{t}\right\|_{L^{2}}\right\} .
\end{aligned}
$$

Using the inequality $a b \leqslant \frac{1}{2}\left(a^{2}+b^{2}\right)$, we get

$$
\begin{aligned}
Q\left(a, E, \psi_{t}\right) \leqslant & C\left\{\|E\|_{L^{2}}^{2}+h^{2}\left\|\varepsilon_{t}\right\|_{L^{2}}^{2}+h^{2}\left(\sum_{i=1}^{N} h_{i}^{2 s}\left\|u_{t}\right\|_{H^{s}\left(I_{i}\right)}^{2}\right)^{1 / 2}\right\} \\
& +\eta h^{2}\left|\left(E_{t}, 1\right)\right|^{2} .
\end{aligned}
$$

Thus, on using (3.21) on (3.20), choosing $\eta$ appropriately, and using the fact that $V^{2}(0, t) \geqslant 0$, we obtain

$$
\begin{aligned}
\frac{1}{2} \frac{d}{d t}\|\sqrt{a} E\|_{L^{2}}^{2} \leqslant & C\left\{\|E\|_{L^{2}}^{2}+h^{2}\left\|\varepsilon_{t}\right\|_{L^{2}}^{2}+h^{2}\left(\sum_{i=1}^{N} h_{i}^{2 s}\left\|u_{t}\right\|_{H^{s}\left(I_{i}\right)}\right)\right\} \\
& +\left(\varepsilon_{t}, \psi_{t}\right)+q\left(u_{t}, \psi_{t}\right),
\end{aligned}
$$


and, on integrating over $t$ from 0 to $\tau$, we get

$$
\begin{aligned}
&\|\sqrt{a} E\|_{L^{2}}^{2}(\tau) \leqslant C\left\{\|E\|_{L^{2}}^{2}(0)+\|E\|_{L^{2}\left(0, \tau, L^{2}\right)}^{2}\right. \\
&+h^{2}\left\|\varepsilon_{t}\right\|_{L^{2}\left(0, \tau ; L^{2}\right)}^{2} \\
&\left.+h^{2}\left(\sum_{i=1}^{N} h_{i}^{2 s}\left\|u_{t}\right\|_{L^{2}\left(0, \tau ; H^{s}\left(I_{i}\right)\right)}\right)\right\} \\
&+2 \int_{0}^{\tau}\left(\varepsilon_{t}, \psi_{t}\right) d t+2 \int_{0}^{\tau} q\left(u_{t}, \psi_{t}\right) d t .
\end{aligned}
$$

Using integration by parts in $t$ on the last two terms, we obtain

$$
\int_{0}^{\tau}\left(\varepsilon_{t}, \psi_{t}\right) d t=\left(\varepsilon_{t}(\cdot, \tau), \psi(\cdot, \tau)\right)-\left(\varepsilon_{t}(\cdot, 0), \psi(\cdot, \tau)\right)-\int_{0}^{\tau}\left(\varepsilon_{t t}, \psi\right) d t
$$

and

$$
\int_{0}^{\tau} q\left(u_{t}, \psi_{t}\right) d t=q\left(u_{t}(\cdot, \tau), \psi(\cdot, \tau)\right)-q\left(u_{t}(\cdot, 0), \psi(\cdot, 0)\right)-\int_{0}^{\tau} q\left(u_{t t}, \psi\right) d t .
$$

These terms can be bounded as follows: the first one using Cauchy-Schwarz inequality, (3.17), Corollary 3.1, and the inequality $a b<\frac{1}{2}\left(a^{2}+b^{2}\right)$, to get

$$
\begin{aligned}
& \left|\int_{0}^{\tau}\left(\varepsilon_{t}, \psi_{t}\right) d t\right| \leqslant C\left\{\left\|\varepsilon_{t}\right\|_{H^{-1}}(\tau)\|\psi\|_{H^{1}}(\tau)+\left\|\varepsilon_{t}\right\|_{H^{-1}}(0)\|\psi\|_{H^{1}}(0)\right. \\
& \left.+\left\|\varepsilon_{t t}\right\|_{L^{2}\left(0, \tau ; H^{-1}\right)}\|\psi\|_{L^{2}\left(0, \tau ; H^{1}\right)}\right\} \\
& \leqslant C\left\{h\left(\|\varepsilon\|_{L^{2}}(\tau)+\left\|\varepsilon_{t}\right\|_{L^{2}}(\tau)\right)\|E\|_{L^{2}}(\tau)\right. \\
& +h\left(\|\varepsilon\|_{L^{2}}(0)+\left\|\varepsilon_{t}\right\|_{L^{2}}(0)\right)\|E\|_{L^{2}}(0) \\
& \left.+h\|\varepsilon\|_{H^{2}\left(0, \tau ; L^{2}\right)}\|E\|_{L^{2}\left(0, \tau ; L^{2}\right)}\right\} \\
& \leqslant C\left\{\|E\|_{L^{2}}^{2}(0)+\|E\|_{L^{2}\left(0, \tau ; L^{2}\right)}^{2}+h^{2}\|\varepsilon\|_{H^{2}\left(0, \tau ; L^{2}\right)}^{2}\right\}+\eta\|E\|_{L^{2}(\tau)}^{2} \text {. }
\end{aligned}
$$

Similarly, using Lemma 3.5 and proceeding as above, we get

$$
\begin{aligned}
\left|\int_{0}^{\tau} q\left(u_{t}, \psi_{t}\right) d t\right| \leqslant C\left\{\|E\|_{L^{2}}^{2}(0)\right. & +\|E\|_{L^{2}\left(0, \tau, L^{2}\right)}^{2} \\
& \left.+h^{2}\left(\sum_{i=1}^{N} h_{i}^{2 s}\left\|u_{t}\right\|_{H^{1}\left(0, \tau ; H^{s}(I)\right)}^{2}\right)\right\}+\eta\|E\|_{L^{2}(\tau) .}^{2}
\end{aligned}
$$

Hence, on combining the above estimates and choosing $\eta$ appropriately, we conclude

$$
\begin{aligned}
\|E\|_{L^{2}}^{2}(\tau) \leqslant C\left\{\|E\|_{L^{2}}^{2}(0)+\|E\|_{L^{2}\left(0, \tau ; L^{2}\right)}^{2}\right. & +h^{2}\|\varepsilon\|_{H^{2}\left(0, \tau, L^{2}\right)} \\
& \left.+h^{2}\left(\sum_{i=1}^{N} h_{i}^{2 s}\left\|u_{t}\right\|_{H^{1}\left(0, \tau ; H^{s}(I)\right)}^{2}\right)\right\} .
\end{aligned}
$$

Therefore, from (2.2ii), (3.1), and Gronwall's lemma, we conclude the proof of Theorem 3.3.

Theorem 3.1 follows from Theorem 3.2, Theorem 3.3, and the triangle inequality.

4. Appendix. In this appendix we prove Lemma 2.1. To do this we prove some approximation results for the projection of a function into $\mathfrak{N}_{0}^{r}$ using $\mathfrak{M}_{-1}^{r-1}$ as test 
space. The estimates are contained in Lemmas 4.1 and 4.2. The projection is defined as follows:

Consider a function $v \in H^{s}(I), v(1)=0$, for $s>0$, and let $V \in \mathfrak{N}_{0}^{r}$ satisfy

$$
(v-V, Q)=0, \quad \forall Q \in \mathfrak{T}_{-1}^{r-1} \text {. }
$$

In order to obtain estimates for this projection, we define a local projection which is defined and analyzed in the following lemma.

LEMMA 4.1. Let $v$ be a function on the interval $F=[b, d]$ and $v \in H^{s}(F)$, $1 \leqslant s \leqslant r+1$, and let $Y \in P_{r}(F)$ satisfy

$$
\int_{F}(v-Y) Q d x=0, \quad \forall Q \in P_{r-1}(F),
$$

and $Y(d)=v(d)$. Then, there is a constant $C$, independent of $h$, such that

$$
\|Y-v\|_{L^{2}(F)}+\mu\left\|(Y-v)_{x}\right\|_{L^{2}(F)} \leqslant C \mu^{s}\|v\|_{H^{s}(F)}
$$

and

$$
|(Y-v)(b)| \leqslant C \mu^{s-1 / 2}\|v\|_{H^{s}(F)}
$$

where $\mu=|d-b|$.

Proof. Let $w(x)=d-x$, and define $W$ and $\zeta$ in $P_{r-1}(F)$ by the relations

$$
Y(x)=v(d)+w(x) W(x), \quad \chi(x)=v(d)+w(x) \zeta(x),
$$

where $\chi \in P_{r}(F), \chi(d)=v(d)$. Then (4.2) is equivalent to

$$
\int_{F} w(W-\zeta) Q d x=\int_{F}(v-\chi) Q d x, \quad \forall Q \in P_{r-1}(F) .
$$

Take $Q=W-\zeta$ to get

$$
\int_{F} w Q^{2} d x=\int_{F}(v-\chi) Q d x \leqslant\|v-\chi\|_{L^{2}(F)}\|Q\|_{L^{2}(F)} .
$$

By changing variables from $[b, d]$ to $[-1,1]$ and considering an appropriate Ritz quotient, it follows that there is a constant $C$, independent of $\mu$, such that for $Q \in P_{r-1}(F)$

$$
C \mu\|Q\|_{L^{2}(F)}^{2} \leqslant \int_{F} w Q^{2} d x .
$$

Thus $\mu\|Q\|_{L^{2}(F)} \leqslant C\|v-\chi\|_{L^{2}(F)} ;$ moreover

$$
\|Y-\chi\|_{L^{2}(F)} \equiv\|w Q\|_{L^{2}(F)} \leqslant\|w\|_{L^{2}(F)}\|Q\|_{L^{2}(F)} \leqslant C \mu\|Q\|_{L^{2}(F)} .
$$

Thus

$$
\|Y-\chi\|_{L^{2}(F)} \leqslant C\|v-\chi\|_{L^{2}(F)}
$$

and, by the inverse property (2.10), we get

$$
\mu\left\|(Y-\chi)_{x}\right\|_{L^{2}(F)} \leqslant C\|Y-\chi\|_{L^{2}(F)} .
$$

From the triangle inequality, (4.5), and (4.6) we get (4.3). To obtain (4.4) notice that

$$
\frac{1}{2}(v-Y)^{2}(b)=-\int_{F}(v-Y)(v-Y)_{x} d x \leqslant\|v-Y\|_{L^{2}(F)}\left\|(v-Y)_{x}\right\|_{L^{2}}
$$

from which use of (4.3) gives (4.4). 
In the following lemma we state and prove the approximation results for (4.1).

Lemma 4.2. Let $v \in H^{s}(I), v(1)=0$, and let $V \in \mathfrak{T}_{0}^{r}$ satisfying (4.1). Then, there exists a constant $C$, independent of $h$, such that

$$
\|v-V\|_{L^{2}}+h\left\|(v-V)_{x}\right\|_{L^{2}} \leqslant C h^{s-1}\|v\|_{H^{s}}, \quad 1 \leqslant s \leqslant r+1 .
$$

Proof. The proof demonstrates that the following estimates hold for each subinterval $I_{i}$.

$$
\begin{aligned}
\|v-V\|_{L^{2}\left(I_{i}\right)}+ & h_{i}\left\|(v-V)_{x}\right\|_{L^{2}\left(I_{i}\right)} \\
& \leqslant C\left\{h_{i}^{s}\|v\|_{H^{s}\left(I_{i}\right)}+h_{i}^{1 / 2}\left(\sum_{j=i+1}^{N} h_{j}^{s-1 / 2}\|v\|_{H^{s}\left(I_{j}\right)}\right)\right\}
\end{aligned}
$$

and

$$
\left|(v-V)\left(x_{i}\right)\right| \leqslant C\left(\sum_{j=i}^{N} h_{j}^{s-1 / 2}\|v\|_{H^{s}\left(I_{j}\right)}\right) .
$$

The lemma follows readily from (4.7) and (4.8). Notice that these estimates clearly demonstrate that the "pollution" effect as one proceeds from the right to the left of the interval remains bounded at the level of the discretization error; therefore, the loss of a power in the estimate.

Take $Q \in P_{r-1}\left(I_{N}\right)$ in (4.1), then by Lemma 4.1 these estimates hold. We now assume that (4.7) and (4.8) hold for $I_{k+1}, \ldots, I_{N}$, and we show that they hold for $I_{k}$.

Let $W \in P_{r}\left(I_{k}\right)$ satisfy $W\left(x_{k+1}\right)=v\left(x_{k+1}\right)$ and

$$
\int_{I_{k}}(W-v) Q d x=0, \quad \forall Q \in P_{r-1}\left(I_{k}\right) .
$$

Thus, by Lemma 4.1,

$$
\|(W-v)\|_{L^{2}\left(I_{k}\right)}+h_{k}\left\|(W-v)_{x}\right\|_{L^{2}\left(I_{k}\right)} \leqslant C h_{k}^{s}\|v\|_{H^{s}\left(I_{k}\right)}
$$

and

$$
\left|(W-v)\left(x_{k}\right)\right| \leqslant C h_{k}^{s-1 / 2}\|v\|_{H^{s}\left(I_{k}\right)} .
$$

Using (4.1) and (4.9), we get

$$
\int_{I_{k}}(W-V) Q d x=\int_{I_{k}}(v-V) Q d x=0, \quad \forall Q \in P_{r-1} .
$$

Thus $(W-V)(x)=C_{r} L_{r}(x)$, where $L_{r}(x)$ is the Legendre polynomial of degree $r$ on the interval $I_{k}$ and

$$
C_{r}=\left(v\left(x_{k+1}\right)-V\left(x_{k+1}\right)\right) / L_{r}\left(x_{k+1}\right) .
$$

Thus, by our assumption that (4.8) holds for $I_{k+1}$, we get

$$
\begin{aligned}
\|W-V\|_{L^{2}\left(I_{k}\right)} & \leqslant\left|C_{r}\right|\left\|L_{r}\right\|_{L^{2}\left(I_{k}\right)} \leqslant C h_{k}^{r+1 / 2}\left|C_{r}\right| \\
& \leqslant C h_{k}^{1 / 2}\left(\sum_{j=k+1}^{N} h_{j}^{s-1 / 2}\|v\|_{H^{s}\left(I_{j}\right)}\right)
\end{aligned}
$$

and $\left|(W-V)\left(x_{k}\right)\right|=\left|(W-V)\left(x_{k+1}\right)\right|$. 
Hence, by the triangle inequality, (4.7) and (4.8) follow. This completes the proof of the lemma.

Corollary 4.1. Let $v$ and $V$ be as in Lemma 4.2. Then, there exists a constant $C$, independent of $h$, such that

$$
\|V\|_{L^{2}} \leqslant C\|v\|_{H^{1}}
$$

We are now ready to give the proof of Lemma 2.1. Let $F \in \Re_{-1}^{r-1}$ and $v \in H^{1}$. Then for $V \in \mathfrak{M}_{0}^{r}$

$$
(F, v)=(F, v-v(1)-V)+(F, v(1))+(F, V) .
$$

Take $V \in \Re_{0}^{r}$ to satisfy

$$
(\chi, v-v(1)-V)=0, \quad \forall \chi \in \mathfrak{T}_{-1}^{r-1} .
$$

Hence, by Corollary $4.1,\|V\|_{L^{2}} \leqslant C\|v\|_{H^{1}}$. Thus

$$
\begin{aligned}
\frac{(F, v)}{\|v\|_{H^{1}}} & \leqslant \frac{|(F, v(1))|}{\|v\|_{H^{1}}}+C \frac{|(F, V)|}{\|V\|_{L^{2}}} \\
& \leqslant|(F, 1)| \frac{|v(1)|}{\|v\|_{H^{1}}}+C \sup _{0 \neq \chi \in \mathscr{R}} \frac{|(F, \chi)|}{\|\chi\|_{L^{2}}},
\end{aligned}
$$

which proves the lemma.

Acknowledgements. The authors wish to thank Professors Graeme Fairweather of the University of Kentucky, Todd Dupont of the University of Chicago and the referee for their helpful comments.

DREM, Suite 620

4151 South West Freeway

Houston, Texas 77027

Department of Mathematics

University of Kentucky

Lexington, Kentucky 40506

1. D. ARCHER, "Collocation in $C^{0}$ spaces for first order hyperbolic equations. I: Optimal order global estimates for quasilinear problems," SIAM J. Numer. Anal., v. 15, 1978, pp. 271-281.

2. G. A. BAKer, “A finite element method for first order hyperbolic equations," Math Comp., v. 29, 1975, pp. 995-1006.

3. J. C. Diaz Velasco, Ph. D. Thesis, Rice University, Houston, Texas, 1974.

4. J. C. DiAz, "A collocation-Galerkin method for the two point boundary value problem using continuous piecewise polynomial spaces," SIAM J. Numer. Anal., v. 14, 1977, pp. 844-858.

5. J. C. DiAz, "Collocation $H^{-1}$-Galerkin method for parabolic problems with time dependent coefficients," SIAM J. Numer. Anal., v. 16, 1979, pp. 911-922.

6. R. J. DUNN, JR. \& M. F. WheELER, "Some collocation-Galerkin methods for two-point boundary value problems," SIAM J. Numer. Anal., v. 13, 1976, pp. 720-723.

7. M. F. WHEELER, “An elliptic collocation-finite element method with interior-penalties," SIAM J. Numer. Anal., v. 15, 1978, pp. 152-161. 